\title{
Uso y apropiación de la tecnología de información y comunicación:đos conceptos para la negociación internacional en organizaciones productivas
}

FECHA DE RECEPCIÓN: 23 de agosto FECHA DE APROBACIÓN: 17 de octubre Pp. 56-69

Sara Aguilar Barrientos M.* Luis Fernando Bustamante $Z_{\text {. ** }}$ José Cano A.***

The appropriation and use of communication and information technologies: two concepts for international trade in the production sector

Utilisation et appropriation des technologies de l'information et de la communication : deux concepts de négociatín internationale pour les organisations

Uso e apropriaçãa da tecnologia da informaçã e comunicaçãa: dois conceitos para a negociação internacional em organizaçũes produtivas
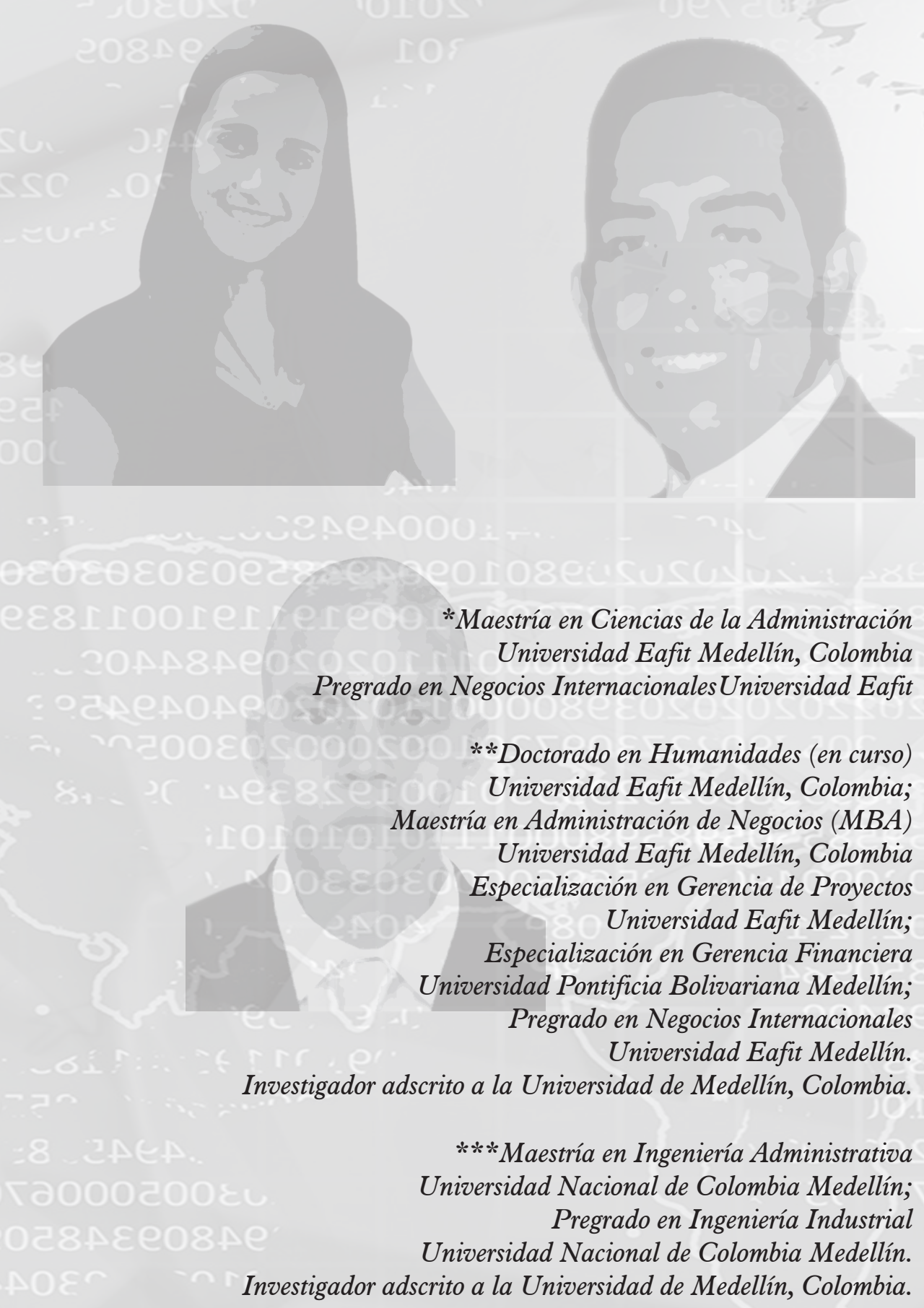


\section{RESUMEN}

El presente artículo, de reflexión pretende aclarar los conceptos de uso y apropiación de las tecnologías de información y comunicación en los procesos de negociación internacional de las organizaciones productivas. Para esto, se realizó un recorrido bibliográfico consignando las principales acepciones de los términos, integrados en una reflexión desde perspectivas semánticas y administrativas. Finalmente, se discute su significado en la práctica para argumentar que la realidad de la empresa se centra en el uso, en vez de la apropiación de TIC para la negociación internacional de las organizaciones productivas. Sobre este aspecto quedan amplios horizontes de investigación.

\section{ABSTRACT}

This article aims at clarifying the concepts of appropriation and use of information and communication technologies in international negotiation processes made by productive organizations. For this, a bibliographic revision was made collecting the meaning of terms, integrated into an integral reflection method based on semantic and administrative perspectives. Finally, we discuss its empirical meaning to affirm that the real essence of the company focuses on the use, rather than on the appropriation of ICTs for the international negotiations done by productive organizations, in which huge research horizons remain accessible for future research.

\section{RESUMÉÉ}

Cet article vise à clarifier les concepts d'utilisation et d'appropriation des technologies de l'information et de la communication dans les processus de négociations internationales des organisations. Pour cela, une analyse bibliographique a été réalisée reprenant les principaux sens des termes utilisés et intégrant une réflexion sémantique et administrative de leur usage. Leur sens d'utilisation pratique permet de déduire que les entreprises se centrent davantage sur l'utilisation que sur l'appropriation des TIC lors de leurs processus de négociation internationale.

\section{RESUMO}

Este artigo de reflexão pretende esclarecer os conceitos de uso e apropriação das tecnologias de informação e comunicação nos processos de negociação internacional das organizações produtivas. Para este propósito realizou-se uma revisão bibliográfica coletando as principais acepções dos termos, integrados em uma reflexão desde perspectivas semânticas $e$ administrativas. Finalmente, discutiu-se seu significado na prática para argumentar que a realidade da empresa está centrada no uso, em vez da apropriação de TIC para a negociação internacional das organizações produtivas. Neste campo ainda há amplos horizontes de pesquisa.

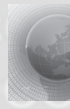

\section{Palabras claves}

Tecnologías de información y comunicación

Negociación internacional

Organizaciones productivas

Uso y apropiación

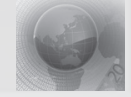

Key words

Information and communication technologies International negotiation productive organizations Se and appropriation

Technologies de l'information et de la Communication Négociation internationale Organisations productives Utilisation et appropriation

\section{Mots clefs}

Palavras-chave

Tecnologias de informação e comunicação

Negociação internacional

Organizações produtivas

Uso e apropriação 


\section{INTRODUCcIóN}

L a competitividad de la organización productiva contemporánea está definida por diversos factores, entre ellos, las Tecnologías de Información y Comunicación (TIC) necesarias para afrontar los retos de la globalización. Sin embargo, como afirmó Fayol (1974) y posteriormente Sallenave (2002), la dirección es clave en la articulación de todas las herramientas para convertirlas en verdaderas fuentes de habilidades distintivas, construyendo la estructura empresarial hacia la generación de valor agregado, único objetivo de la verdadera planeación estratégica (Hill y Jones, 2000). En otras palabras, si la existencia de las TIC en las empresas, no se traduce en la generación de habilidades distintivas que llevan a ventajas competitivas, probablemente se estén convirtiendo en una carga adicional para la empresa en internacionalización.

Por lo anteriormente mencionado, las TIC pueden pensarse como mecanismos para expandir las funciones de la empresa más allá de los bordes domésticos y por tanto, son indispensables en la negociación internacional. Sea que se hable de un enfoque de integración o de distribución (Merlano, 1994), la negociación internacional responde a la necesidad de conformar redes en el exterior. Es necesario introducir esta problemática a la empresa en internacionalización, aquella que no escapa a los procesos de globalización y por ende, afronta desafíos más complejos (Deresky, 2005), como es el caso de Colombia (Ghauri y Usunier, 2003). Ciertamente, las empresas en internacionalización requieren el uso de herramientas que faciliten su supervivencia en el entorno internacional (Ball y McCulloch, 2003), para superar la transformación de las funciones de la empresa ${ }^{1}$.

Esto significa que la respuesta a la complejidad de la empresa en internacionalización, no está en la herramienta o Software usado, sino la forma en que se use durante los procesos que generan los vínculos entre la empresa y el entorno internacional, es decir, la negociación (Starkey,
Boyer y Wilkenfeld, 2005). Por otro lado, el proceso de negociación (Fisher, Ury, y Patton, 1991), requiere de la mediación de herramientas bien aprovechadas, ya que estas no sólo afectan el resultado de los procesos, sino los comportamientos de las personas involucradas en la negociación (Thompson y Nadler, 2002).

La evidencia teórica sugiere que la forma en que se usen las TIC, tiene repercusiones en el desempeño de las organizaciones productivas (Alvadbi, Keramati, y Razmi, 2007). También hay estudios que demuestran que esta relación no es posible de comprobar, aunque los gerentes reconocen en la gestión de la información y las herramientas asociadas, la base para la construcción, diseminación y aplicación del conocimiento organizacional (Senge, 2004). Lamentablemente, las relaciones entre el ser humano y la tecnología han obligado a privilegiar las herramientas sobre el hombre, situación que no permite que los beneficios de las TIC sean correctamente aprehendidos en las organizaciones. Es así, como algunas estructuras empresariales se configuran alrededor de herramientas tecnológicas en detrimento del talento humano, puesto que no se acompañan de procesos de aprendizaje organizacional durante la implementación (García, 1997).

En concordancia con el problema planteado en términos del uso y la apropiación de las TIC en las organizaciones productivas para los procesos de negociación internacional, este artículo de reflexión se basa en una perspectiva interdisciplinar, en las concepciones más importantes sobre tecnología de comunicación, tecnología de información, y tecnología de información y comunicación. Luego, teniendo este marco en mente, se presentará parte del método que guió el desarrollo de esta investigación y dio vida a este producto. Posteriormente, se aclararán los conceptos de uso y apropiación de tecnología como parte de los resultados de una visión analítica, interpretativa y crítica de los autores.

\footnotetext{
${ }^{1}$ Si las vemos de cualquier manera o como Fayol (1974) propuso: técnica, comercial, financiera, seguridad, contabilidad y administrativa.
} 


\section{MARCO TEÓRICO}

\subsection{Tecnología}

La tecnología se considera como el "conjunto de teorías y de técnicas que permiten el aprovechamiento práctico del conocimiento científico" (RAE, 2012). Bajo este punto de vista, Derry y Williams (1960) sostienen que la tecnología comprende todo el cuerpo del conocimiento y los dispositivos por medio de los cuales el hombre progresivamente ha ido dominando y reinando sobre el ambiente natural que lo rodea. Drucker (2011), comenta que la tecnología no consiste en herramientas, sino que abarca la forma de cómo el ser humano trabaja. Esto se debe a que las instituciones y organizaciones son vistas como una extensión del hombre, y la tecnología puede entenderse como la forma más efectiva en la cual se ejecuta la labor. Desde el punto de vista de organizaciones productivas, la tecnología puede ser vista como explica la tabla 1.

Tabla 1. Definiciones de tecnología

\begin{tabular}{|l|l|}
\hline \multicolumn{1}{|c|}{ Autor } & \multicolumn{1}{c|}{ Definición } \\
\hline Sanchis (2011) & $\begin{array}{l}\text { El estudio de las aplicaciones técnicas } \\
\text { hacia objetos y hombres dentro de una } \\
\text { actividad productiva específica y es el } \\
\text { conocimiento sistemático de los procesos } \\
\text { industriales y su aplicación }\end{array}$ \\
\hline $\begin{array}{l}\text { Collin, Collin, y } \\
\text { Collin (2002) }\end{array}$ & $\begin{array}{l}\text { La aplicación del conocimiento científico } \\
\text { a procesos industriales }\end{array}$ \\
\hline RAE (2012) & $\begin{array}{l}\text { "Conjunto de los instrumentos y proce- } \\
\text { dimientos industriales de un determinado } \\
\text { sector o producto" }\end{array}$ \\
\hline $\begin{array}{l}\text { Kogut y Zander } \\
\text { (1992) }\end{array}$ & $\begin{array}{l}\text { El conocimiento por medio del cual se } \\
\text { crea y produce un producto }\end{array}$ \\
\hline
\end{tabular}

Fuente. Elaboración propia de los autores.

Los avances tecnológicos acompañaron la evolución de la humanidad y la civilización, siendo resultado de una construcción multicultural colectiva a lo largo de la historia (Pacey, 1991). Además transforma el entorno entre otros, la guerra, nuevos estilos de vida, revoluciones estructurales, industriales (Kranzberg y Pursell, 1967; Derry y Williams, 1960) donde se incluye la revolución tecnológica que se basa fuertemente en el conocimiento por medio de productos digitales, automatización y la Internet para modificar la organización de la sociedad y de las unidades productivas (Yoguel, 2008; Alierta, 2011). Esta revolución tecnológica, experimenta fases de instalación, reacomodación y declinación, haciendo que la posición estratégica de empresas, industrias y países enteros, cambie. De esta forma, la tecnología ha permitido cerrar algunas

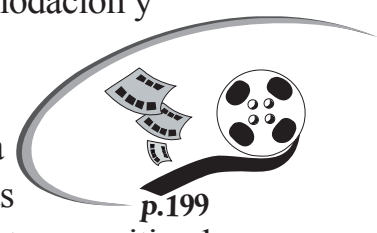
brechas socioeco-nómicas y de aislamiento, permitiendo que regiones en vía de desarrollo, se abran hacia la modernidad (Alierta, 2011).

La aparición de nuevas tecnologías crea la necesidad de hablar de Tecnologías de Información y Comunicación, dividiendo el concepto inicialmente en Tecnologías de Información y Tecnologías de Comunicación o Telecomunicaciones.

\subsection{Tecnologías de información}

El término de Tecnologías de Información (TI), fue utilizado por primera vez por Leavitt y Whisler (1958), quienes describían que las TI se componían de varias partes relacionadas, tales como: técnicas para el procesamiento rápido de grandes cantidades de información que se materializan en computadores de alta velocidad, métodos matemáticos y estadísticos para la toma de decisiones, como la programación lineal y la investigación de operaciones y simulación de razonamientos a través de programas de computador. De acuerdo con la Information Technology Infrastructure Library (ITIL), hoy en día las TI representan "el uso de la tecnología para el almacenamiento, la comunicación o el procesamiento de la información, (...) la información tratada puede incluir datos del negocio, voz, imágenes, vídeo, etc." (ITIL, 2011, p. 56); se encargan de adquirir, almacenar, procesar y distribuir información en medios electrónicos, como por ejemplo la radio, televisión, teléfono y computadores (Collin, Collin, y Collin, 2002), 
agregando valor a las organizaciones al crear rutinas lógicas de decisión y gestionar datos extensos y complejos (Davenport y Short, 1990).

Para Orlikowski y Robey (1991), las TI son antecedente y consecuencia de las acciones de las organizaciones productivas, debido a que las TI pueden ser producto de la acción humana cuando estas dan en contextos culturales y estructurales específicos y también, pueden ser un medio de la acción humana cuando facilitan y limitan las acciones a través de esquemas de interpretación, normas, reglas y recursos que contribuyen a la creación, recreación y transformación de contextos culturales y estructurales específicos.

Una de las principales razones para usar TI en las organizaciones productivas, es que facilitan la tarea de diseñar, implementar y controlar procesos además de aumentar la capacidad de soportar actividades interpersonales por medio de desarrollos orientados a redes basadas en correos electrónicos, conferencias, herramientas de lluvias de ideas, entre otros; lo anterior desencadena la creación de capacidades transaccionales, geográficas, automáticas, informáticas, de secuencia, de gestión del conocimiento, de rastreo y de desintermediación en las organizaciones productivas (Davenport y Short, 1990). Esto hace que las TI, ofrezcan potencial de mejorar el desempeño administrativo de una organización (Davis, 1989), especialmente cuando se utiliza la información para mejorar productos y procesos (Zuboff, 1988) y cuando se descentraliza la toma de decisiones para utilizar las TIC en el apoyo de los procesos de negocio (Applegate, Cash Jr, y Mills, 1988; ITIL, 2011).

\subsection{Tecnologías de comunicación (TC)}

La Tecnología de Comunicación, o telecomunicación, es aquella cuyo fin es emitir, transmitir y recibir signos, señales, escritos, sonidos, datos o información de cualquier naturaleza, a distancia a través de hilo, ondas de radio, medios visuales u otros sistemas electromagnéticos, que se materializan en medios como el teléfono, satélites, radio y otros, capaces de llevar señales. Estas tecnologías, deben permitir el intercambio de información entre dos individuos y fomentar el desarrollo de la percepción, expectativas y participación que conforman los fundamentos de la comunicación (Drucker, 2011).
Para evaluar la importancia que tienen las telecomunicaciones en el desarrollo económico de los países, Roller y Waverman (2001) desarrollaron una investigación donde encontraron, con aclaraciones y salvedades, que en general hay una relación positiva con la producción y crecimiento económico cuando existe una masa crítica de infraestructura de telecomunicaciones. Así mismo, en un reciente estudio de Hilbert y López (2011), se destaca que la capacidad mundial de telecomunicaciones ha estado creciendo desde los años 80 y que han sido dominadas por las tecnologías digitales desde 1990. En procesos de negociación entre organizaciones productivas, existen medios de comunicación tales como el teléfono, fax, $E$-mail, Pager o Beeper, voz sobre protocolo de Internet (Voz IP VoIP), los cuales ayudan a que dichos procesos se lleven a cabo de forma satisfactoria (Low y Ang, 2011).

\subsection{Tecnologías de Información y Comunicación (TIC)}

De acuerdo con Schiavo (2007), se puede definir a las TIC como las tecnologías desarrolladas a partir de la electrónica, microchips y computadoras personales, que permitieron la creación de la Internet como resultado de la convergencia entre las tecnologías de informacióny las telecomunicaciones. Dichas tecnologías, son capaces de producir, almacenar y transmitir información digital y de funcionar en red como plataforma organizativa de las diversas actividades humanas y sociales que soportan. Para Correa, Gómez, y Cano (2010), las TIC son el estudio, diseño, desarrollo, fomento, mantenimiento y administración de la información por medio de sistemas informáticos, esto no solamente incluye la computadora, el medio más versátil y utilizado, sino también los teléfonos celulares, la televisión, la radio, los periódicos digitales, etc. De esta forma, las TIC son la evolución del conjunto de herramientas de la

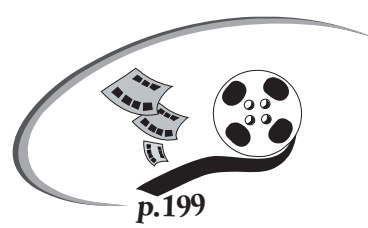
información y comunicación que se incorporan en las organizaciones y actores sociales para la circulación de información, tanto en áreas de gestión y administración, como en la producción de bienes y servicios y se pueden manifestar como capacidades ofrecidas por los computadores, aplicaciones de Software y telecomunicaciones (Yoguel, 2008). 
Alierta (2011) agrupa tres categorías de impactos de las TIC: impactos económicos (aumento de efectividad y eficiencia de las tareas y procesos que se llevan a cabo en las organizaciones productivas que repercute en la evolución de productividad para una economía), impactos en los negocios (mejoramiento de la eficiencia, productividad y competitividad dentro de las empresas), e impactos sociales (nuevas formas de interacción entre individuos, grupos y empresas, facilitando la cooperación y eliminación de barreras de espacio, tiempo y lugar).

La investigación, desarrollo e innovación en las TIC se ha enfocado en dar respuesta a retos socioeconómicos, de interés y alcance mundial, y de igual forma, juega un papel fundamental en el avance e innovación de sectores y productos (Vickery y Wunsch-Vincent, 2009), lo cual destaca que las TIC pueden generar impactos significativos en la competitividad de las organizaciones productivas cuando se garantizan cambios que se enfoquen en facilitar su apropiación; es decir, las TIC por sí solas no mejoran las organizaciones y deben ser observadas como un apoyo a los procesos empresariales. Para garantizar que la incorporación de TIC desarrolle ventajas competitivas en las organizaciones se requiere contar con habilidades y competencias endógenas o internas que permitan y faciliten la creación, transmisión y apropiación de la información difundida por dichas TIC; y de esta forma las TIC junto con la gestión del conocimiento $\mathrm{y}$ los procesos de aprendizaje han permitido generar ventajas competitivas en países desarrollados (Yoguel, 2008).

Es de destacar que las TIC se han desarrollado en el tiempo con base en requerimientos particulares de las organizaciones; y en el ámbito de organizaciones productivas, empresas como Amazon.com e Inditex, y algunos sectores industriales son ejemplo de modelos exitosos de negocio que se han apoyado en TIC para crear ventajas competitivas. (Alierta, 2011).

\subsection{Las TIC en la negociación internacional}

En el campo de las organizaciones productivas, los gerentes reconocen en las TIC una ayuda indispensable para el proceso de negociación. Existe diversa documentación sobre su uso en las empresas y las diferentes potencialidades para la efectividad del proceso negociador. Con el desarrollo de las TIC, las barreras geográficas y la distancia son menos importantes para estar en contacto con otras personas, lo cual genera tanto procesos de descentralización

(existe contacto con partes separadas geográficamente a nivel global), como de centralización (no se requiere tener disperso

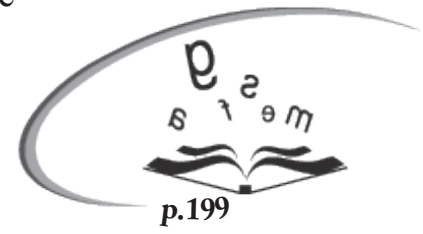
geográficamente a un equipo de trabajo para tener impacto global), permitiendo que los ámbitos local y global puedan ser compatibles (Yoguel, 2008).

Como consecuencia de lo anterior, se ha presentado un crecimiento del comercio B2B (empresa a empresa), basado en Internet, el cual es resultado del aumento del uso de computadores y tecnologías de comunicación en procesos como la negociación ${ }^{2}$. Ya sea por $E$-mail, mercados virtuales $\mathrm{u}$ otra herramienta, esto ayuda a que los negociadores puedan establecer relaciones con cualquier persona en el mundo; dichas relaciones pueden fortalecerse más con herramientas que permitan humanizar la comunicación, tales como la teleconferencia (Low y Ang, 2011).

Aunque el uso de la tecnología de Software no es una condición previa para llevar a cabo las negociaciones, en la economía de la información, las TIC juegan un papel especial en el comercio y por lo tanto, en las negociaciones genera retos y oportunidades para que los gerentes negocien en línea en vez de desplazarse y ubicarse en diferentes lugares. El desarrollo de un buen número de sistemas de información para la negociación, en cuestión de algoritmos, gracias a diferentes investigadores y desarrolladores de Software, ha ampliado las opciones de las TIC tradicionales como el computador, el E-mail, fax, teleconferencias, entre otros (Low y Ang, 2011). La rapidez de adopción de tecnologías como la telefonía móvil, el Internet y el ancho de banda, generan cambios de paradigmas tecnológicos; debido a esto, hoy en día el progreso no existe para aquellos que no están en capacidad de acceder a la intercomunicación global por medio de las TIC (Alierta, 2011).

\footnotetext{
${ }^{2}$ De igual forma, otros esquemas como B2C (empresa a cliente), C2B (cliente a empresa) y C2C (cliente a cliente), se han sofisticado con el tiempo (Cullen y Parboteeah, 2009).
} 


\section{MÉTODO}

S iguiendo los lineamientos para la investigación documental de Hoyos (2000), se ejecutó entre enero y junio de 2012 un recorrido bibliográfico en libros y revistas especializadas en bibliotecas y bases de datos electrónicas que respondieran los términos de búsqueda de negociación y Tecnologías de la Información y la Comunicación.

La información bibliográfica se sistematizó con el uso de formatos de resumen de documentos y fue organizada en tablas analíticas de resumen de contenidos (Miles y Huberman, 1994) para diferenciar los conceptos de uso y apropiación de la tecnología. Lo anterior, con el propósito de soportar el supuesto principal de esta investigación: las
TIC son usadas pero poco apropiadas en los proceso de negociación en organizaciones productivas de Medellín.

Como la idea es seguir un presupuesto constructivista y el desarrollo de un ejercicio que triangula analizando y sintetizando (Bunge, 2000), la información levantada se contrasta con la teoría administrativa y de negociación, especialmente la que alude a la importancia de las TIC para la eficiencia organizacional y el establecimiento de vínculos comerciales por medio de la negociación, donde la aplicación de herramientas tecnológicas es apropiada y pertinente.

Siguiendo las guías metodológicas descritas, se presentan los siguientes resultados a modo de reflexión y conclusiones, preliminares a la culminación del proyecto de investigación al cual está adscrito este artículo.

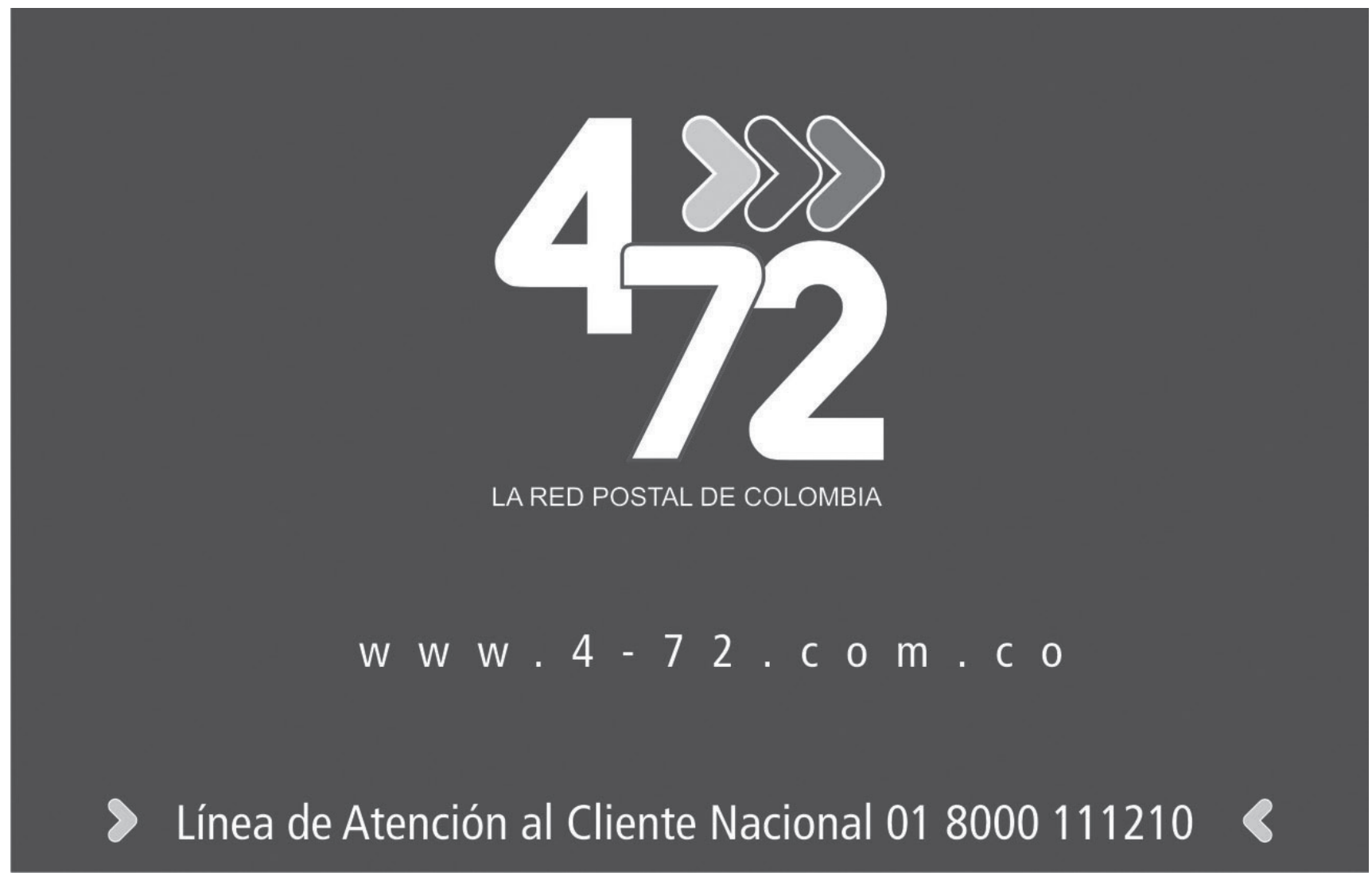




\section{RESULTADOS}

E 1 diccionario de la Real Academia Española, señala que el verbo usar se refiere a "ejercer o servir un empleo u oficio" (RAE, 2012). Con el propósito de diferenciar uso y apropiación, se consultó el significado de la primera en el diccionario de filosofía de Mora (1994), de dónde pueden desprenderse algunas características diferenciadoras.

De acuerdo con su significado, el uso es desprevenido es decir, no persigue un objetivo en particular. Por esto se mencionan dos características esenciales del uso, las cuales aluden a las cualidades de inevitable e inauténtico. Esta última característica, deja entrever que el uso no está "acreditado de cierto y positivo por los caracteres, requisitos o circunstancias que en ello concurren" (Ferrater, 1994), es decir, que no responde a los propósitos específicos que se tengan al realizar una tarea y por tanto, no es fiel a sus orígenes y convicciones.

En la lingüística, se precisa que estudiar los usos de un término no equivale a estudiar las características lingüísticas del mismo. Igualmente, con esta analogía vale la pena clarificar que estudiar los usos que se hace de las Tecnologías de Información y la Comunicación (TIC) no es lo mismo que estudiar las características, aplicaciones y soportes.

Por su parte, la apropiación es prevenida y definida por una intencionalidad. El Diccionario de la Real Academia Española (2012), define el término como "aplicar a cada cosa lo que le es propio y más conveniente". Con esto, quiere decirse que cuando las TIC son adquiridas y puestas en marcha con el objetivo de hacer más eficiente o más simple un procedimiento, o porque las circunstancias así lo requieren para alcanzar mayores y mejores resultados, se está haciendo apropiación. Por lo anterior, puede aseverarse que la apropiación se refiere a las formas de uso que las tecnologías adoptan en su relación con las personas. Por ende, a la acción de usar se suma una intencionalidad y un objetivo, aspecto que se pretende poner de manifiesto en este documento.

De Gortari (1988, p. 549), define uso como el "acto o el modo de adoptar medios, instrumentos o utensilios". Si bien el término tiene cuna en la filosofía, en el ámbito administrativo, las TIC son consideradas medios en las organizaciones productivas para alcanzar efectividad en los procesos internos, que deviene en mayor beneficio económico. Otra noción de uso en contraposición a la de apropiación, está ligada con las ideas de Marx y su teoría del valor (Aguilar, 2012) (Marx, 1974 , pág. 3$)^{3}$, al considerar las nuevas tecnologías como bienes puestos al servicio de las personas.

Es importante resaltar, que lo que se pone en cuestión es justo la apropiación que las personas hacen de las TIC o las formas de uso en los procesos de negociación internacional, no las nuevas tecnologías en sí mismas. Drucker (1997, p. 84), expone esta afirmación y subraya la influencia del hombre sobre la máquina, la cual está de alguna manera subordinada a sus designios: "El trabajador de conocimientos bien puede necesitar una máquina, sea un computador, un analizador de ultrasonido o un telescopio. Pero la máquina no le dice qué hacer ni mucho menos cómo hacerlo".

La apropiación también puede entenderse como la actualización de lo virtual. Lévy (1999, p. 56), expresa que "el bien virtual plantea un problema, abre un campo de interpretación, de resolución o de actualización". En otras palabras, el objeto tecnológico y sus sistemas de comunicación e información, tienen múltiples aplicaciones para ser utilizadas por el hombre; con respecto a esta última, "cuando utilizo la información ${ }^{4}$, es decir, cuando la interpreto, la relaciono con

\footnotetext{
3 En ella, se define valor de uso y valor de cambio, afirmando el primero como la utilidad que un objeto puede brindarle a una persona, sin que esto signifique un intercambio monetario para obtenerlo.

4 Yoguel (2008) explica que en la literatura existen dos posiciones respecto a la funcionalidad de la información: por un lado, quienes consideran que la información es conocimiento y que el acceso a TIC es clave para desarrollar competencias tecnológicas (Cowan y Foray, 1997; Cowan, David, y Foray, 2000), mientras que otros autores argumentan que se requiere desarrollar capacidades previas, para que luego las TIC puedan ser funcionales para el desarrollo de procesos de aprendizaje en una organización (Cimoli, 2000).
} 
otras informaciones para darle sentido o me sirvo de ella para tomar una decisión, la actualizo" (1999, p. 55). Lo mismo ocurre con la comunicación: los medios para comunicarse con el otro son usados por el hombre por diferentes razones, cuando esta apropiación sucede, ella se actualiza.

En este orden de ideas, puede observarse que la virtualización hace parte de la organización y su expansión, pone en evidencia cambios en la forma de ejecutar la acción empresarial (Sallenave, 2002). Con la virtualización, se pasa de una solución dada, al planteamiento de nuevos retos en la administración, puesto que según Lévy (1999), lo virtual se puede asimilar a un problema y lo actual, a una solución. En este sentido, Drucker (1997, p. 73) complementa anunciando que la empresa "tiene que organizarse para el cambio constante" y por supuesto, para la administración renovada por la herramientas que apropia.

No sorprende entonces la utilización que Drucker hace de la palabra uso, ya que invita a encontrar el sentido de la acción empresarial en los propósitos de la empresa:
Los especialistas en información son fabricantes de herramientas. Los usuarios de información, sean ejecutivos o profesionales, tienen que decidir qué información usar, para qué usarla y cómo usarla. [...] El segundo gran reto que enfrentan hoy los usuarios de información es cómo obtener esos datos; cómo ponerlos a prueba; cómo juntarlos con el sistema de información existente para hacerlos eficaces en el proceso decisorio de una compañía (Drucker, 1997, pp. 106-108).

Hay que entender que las TIC existen para servir a las personas que hacen parte de las empresas en la ejecución de tareas, funciones y procesos, pero en las formas de uso, hay tergiversaciones que atentan con su buen funcionamiento. Esto se debe a que los actos humanos y especialmente, en la negociación, están muy lejanos de ser perfectos: hay hábitos de uso de los objetos y sistemas tecnológicos que no necesariamente sirven a los objetivos empresariales, dificultando la apropiación de las herramientas. 


\section{CONCLUSIONES}

E 1 ser humano es el común denominador entre la negociación, la gestión del conocimiento y la gestión de las TIC. La negociación como proceso de comunicación es propia del hombre; la gestión del conocimiento vista como la transformación del conocimiento tácito en explicito, parte de creaciones humanas (Dalkir, 2011) y la gestión de las TIC, según Davenport y Prusak (1999), pone a las personas, no a la tecnología, en el centro del mundo de la información. Es por tanto necesario enfocar la administración de la información y del conocimiento hacia las personas en cuanto son ellas proveedores, procesadores y usuarias de estas.

En esta misma línea, ocurren dos situaciones que limitan el impacto beneficioso de las TIC en el desempeño organizacional: primero, se considera TIC únicamente a aquellas herramientas mediadas por Software y segundo, la organización se modifica para el uso del Software y no para la agregación de valor, desembocando en una situación que altera los objetivos estratégicos de las empresas, en cuanto la agregación de valor es necesaria en el desarrollo de una ventaja competitiva (Hill y Jones, 2000). Por supuesto, ambas son consecuencias de la concepción de uso de tecnología.

La primera situación, en cuanto a la idea sesgada sobre qué es tecnología, ignora el sentido amplio del concepto como "conocimiento del mundo representado en máquinas, personas, planos, procesos" (García,1997, pp.277); desemboca en la segunda situación mencionada, en la cual la tecnoestructura genera presiones para la estandarización de las tareas y supera a los otros componentes de la organización, dando como resultado una burocracia maquinal (Mintzberg, 1992; 1979)5. Entonces, para el funcionamiento adecuado de la empresa, se invierte en Software que se espera solucione todos los problemas, cuando la realidad es que este sólo soluciona los problemas por los cuales se le indague.

Entre las muchas variables que pueden influir en el uso y aceptación de un sistema, pueden existir dos factores determinantes que son de especial importancia: en primer lugar, las personas tienden a utilizar o no, un aplicativo informático de acuerdo a si creen que les ayudará o no a hacer mejor su trabajo, lo que se denomina como utilidad percibida $^{6}$. Y en segundo lugar, cuando los usuarios potenciales de una aplicación informática creen que esta va a serles útil es posible que al mismo tiempo, crean que dichos sistemas sean demasiado difíciles de usar. Una aplicación informática desde el punto de vista de los usuarios, tiene más aceptación de uso cuando se percibe que es más fácil de usar que otra aplicación (Davis, 1989) ${ }^{7}$. En la negociación internacional, es claro que la comunicación ocupa un punto central y por ende, una herramienta que implique su mejoramiento, es pertinente (Kremenyuk, 2002).

Seleccionar las TIC adecuadas, es un proceso crítico dentro de las organizaciones, ya que sobredimensionar o subvalorarlas implica costos y pérdidas de oportunidades. En el manejo de TIC, se debe tomar decisiones respecto a determinar las aplicaciones más apropiadas para mejorar el desempeño de los procesos empresariales y elegir el modelo de gestión que se adapte a las características de cada empresa en particular. Aparte de esto, las empresas requieren capacidad de gestión de tecnología para identificar qué contribuciones realizadas por las TIC, son pertinentes en la satisfacción de las necesidades específicas de la organización (Alierta, 2011).

\footnotetext{
5 De acuerdo con Mintzberg (1979), la estructura de las organizaciones se compone de ápice estratégico, ideología, tecnoestructura, línea media, Staff de apoyo y núcleo de operaciones.

6 La utilidad percibida se puede definir como el grado en el cual una persona cree que va a aumentar su desempeño al utilizar un sistema en particular. Esto se desprende de la palabra útil, que significa algo capaz de ser usado de forma ventajosa. Un sistema se percibe de alta utilidad cuando un usuario cree en la existencia de una relación positiva hacia el desempeño (Davis, 1989).

${ }^{7}$ Por otro lado, la facilidad de uso que se percibe de un sistema se refiere al grado en el cual una persona cree que usando un sistema en particular, se liberará a sí mismo de esfuerzos, considerando que los esfuerzos son recursos finitos que una persona asignara a varias actividades de las cuales es responsable (Radner y Rothschild, 1975).
} 


\section{REFERENCIAS}

Aguilar, S. (2012). Relaciones sociales en las organizaciones productivas: implicaciones por el uso de las nuevas tecnologías. España-Alemania: Editorial Académica Española.

Alierta, C. (2011). The Promise of Technology. En S. Dutta, y I. Mia (Eds), The Global Information Technology Report 2010-2011: Transformations 2.0 (pp. 61-68). Geneva, Switzerland: World Economic Forum Editors.

Alvadbi, A., Keramati, A., y Razmi, J. (2007). Assessing the Impact of Information Technology on Firm Performance Considering the Role of Intervening Variables: Organizational Infrastructures And Business Processes Reengineering. International Journal of Production Research, 45(12), 2697-2734.

Applegate, L., Cash Jr, J., y Mills, Q. (1988). Information Technology and Tomorrow's Manager. En W. McGowan (Ed.), Revolution in Real Time: Managing Information Technology in the 1990s (Vol. 66, pp. 128-136). Boston: Harvard Business School Press.

Aristóteles. (2012). Metafisica. Madrid: GREDOS.

Ball, D., y McCulloch, J. W. (2003). Negocios internacionales: introducción y aspectos esenciales. Madrid: McGraw Hill.

Bunge, M. (2000). Buscar la filosofia en las ciencias sociales. México: Siglo XXI.

Cimoli, M. (2000). Networks, Markets Structures and Economic Shocks: the Structural Changes of Innovation Systems in Latin America. Laboratory of Economics and Management (LEM), 46 p.

Collin, S., Collin, P., y Collin, M. (2002). Dictionary of Information Technology (Third ed.). London: Peter Collin Publishing.

Correa, A., Gomez, R., y Cano, J. (2010). Gestión de almacenes y tecnologías de la información y comunicación (TIC). Estudios Gerenciales, 26(117), 145-171.

Cowan, R., y Foray, D. (1997). The Economics of Codification and the Diffusion of Knowledge. Industrial and Corporate Change, 6(3), 595-622.

Cowan, R., David, P., y Foray, D. (2000). The Explicit Economics of Knowledge Codification and Tacitness. Industrial and Corporate Change, 9(2), 211-253.

Cullen, J., y Parboteeah, K. (2009). International Business: Strategy and the Multinational Company. New York: Routledge.

Dalkir, K. (2011). Knowledge Management in Theory and Practice. Cambridge: The MIT Press.

Davenport, T., y Prusak, L. (1999). Ecología de la información: por qué la tecnología no es suficiente para lograr el éxito en la era de la información. México: Oxford University Press. 
Davenport, T., y Short, J. (1990). The new Industrial Engineering. Information Technology and Business Process Redesign. Sloan Management Review, 31(4), 11-27.

Davis, F. (1989). Usefulness, Perceived Ease of Use, and User Acceptance of Information Technology. MIS Quarterly, 13(3), 319-340.

De Gortari, E. (1988). Diccionario de la Lógica. México: Plaza y Valdes.

Deresky, H. (2005). International Management. Managing across Borders and Cultures. New Jersey: Prentice Hall.

Derry, T., y Williams, T. (1960). A Short History of Technology: From the Earliest Times to A.D. 1900. New York: Dover Publications.

Drucker, P. (1997). Su visión sobre: la administración, la organización basada en la información, la economía, la sociedad. Bogotá: Norma.

Drucker, P. (2011). Technology, Management, and Society. Boston: Harvard Business School Publishing.

Fayol, H. (1974). Administración industrial y general. México: Herrero Hermanos Svcs. S.A.

Ferrater, J. (1994). Diccionario de filosofia. Barcelona: Ariel.

Fisher, R., Ury, W., y Patton, B. (1991). Getting to yes: Negotiating Agreement Without Giving in. New York: Penguin Books.

García, A. (1997). La transferencia de tecnología informática: una forma de aprendizaje organizacional. En A. Galvis, y A. Espinosa (Eds.), Estrategia competitividad e informática (pp. 275-284). Bogotá: Uniandes.

Ghauri, P., y Usunier, J. (2003). International Business Negotiations. International business and management series. Oxford: Pergamon.

Hilbert, M., y Lopez, P. (2011). The World's Technological Capacity to Store, Communicate, and Compute Information. Science, 332(6025), 60-65.

Hill, C., y Jones, G. (2000). Administración estratégica: un enfoque integrado. México: McGraw-Hill.

Hoyos, C. (2000). Un modelo para investigación documental. Guía teórico-práctica sobre construcciones de estados del arte. Medellín: Señal Editora.

ITIL. (2011). Glosario y abreviaturas de ITIL Español (Latinoamericano). Recuperado el 27 de Marzo de 2012, de Information Technology Infrastructure Library: http://www.itil-officialsite.com/nmsruntime/saveasdialog.aspx?1ID=1183ysID=242

Kogut, B., y Zander, U. (1992). Knowledge of the Firm, Combinative Capabilities, and the Replication of Technology. Organization Science, 2(3), 383-397. 
Kranzberg, M., y Pursell, C. (1967). Technology in Western Civilization: Technology in the Twentieth Century. New York: Oxford University Press.

Kremenyuk, V. (2002). International Negotiation: Analysis, Approaches, Issues. San Francisco: Jossey-Bass.

Leavitt, H., y Whisler, T. (1958). Management in the 1980. Harvard Business Review, 36(6), 41-41.

Lévy, P. (1999). ¿Qué es lo virtual?. Barcelona: Paidós.

Low, P., y Ang, S. (2011). Information Communication Technology (ICT) for Negotiations. Journal of Research in International Business and Management, 1(6), 183-196.

Marx, K. (1974). El Capital. México: Fondo de Cultura Económica.

Menger, K. (1985). Principios de economía política. Barcelona: Orbis.

Merlano, A. (1994). Aproximación conceptual a los procesos de negociación. Revista Universidad EAFIT, Abril-Junio(94), $17-21$.

Miles, M., y Huberman, M. (1994). Qualitative Data Analysis: An Expanded Source Book. Thousand Oaks: Sage Publications.

Mintzberg, H. (1979). The Structuring of Organizations. Englewood Cliffs, N.J.: Prentice Hall.

Mintzberg, H. (1992). Structure in Fives: Designing Effective Organizations. Upper Saddle. River, NJ: Prentice Hall.

Orlikowski, W., y Robey, D. (1991). Information Technology and the Structuring of Organizations. Information Systems Research, 2(2), 143-169.

Pacey, A. (1991). Technology in World Civilization: A Thousand-Year History. Cambridge: MIT Press.

Radner, R., y Rothschild, M. (1975). On the Allocation of Effort. Journal of Economic Theory, 358-376.

RAE (2012). Diccionario de la Lengua Española- Vigésima segunda edición. Recuperado el 24 de febrero de 2012, de Real Academia Española: http://buscon.rae.es/draeI/SrvltConsulta?TIPO_BUS=3yLEMA=tecnologia.

Roller, L., y Waverman, L. (2001). Telecommunications Infrastructure and Economic Development: A Simultaneous Approach. American Economic Review, 91(4), 909-923.

Sallenave, J. (2002). La gerencia integral. Bogotá: Editorial Norma.

Sanchis, A. (2011). Introducción al pensamiento social de Jacques Ellul. Madrid, España: AEBIUS.

Schivago, E. (2007). Investigación científica y tecnológica en el campo de las TIC: ¿conocimientos técnicos, contextuales o transversales? Revista CTS, 3(9), 91-113. 
Senge, P. (2004). La quinta disciplina: el arte y la práctica de la organización abierta al aprendizaje. Barcelona: Granica.

Starkey, B., Boyer, M., y Wilkenfeld, J. (2005). Negotiating a Complex World: An Introduction to International Negotiation. Oxford: Rowman y Littlefield Publishers.

Thompson, L., y Nadler, J. (2002). Negotiation Via Information Technology: Theory and Application. Journal of Social Issues, 58(1), 109-124.

Vickery, G., y Wunsch-Vincent, S. (2009). RyD Innovation in ICT Sector: Toward Globalization and Collaboration. En W. E. Forum, The Global Information Technology Report (pp. 95-109). Geneva, Switzerland: World Economic Forum Editors.

Yoguel, G. (2008). Información y conocimiento: las vinculaciones entre difusión de TIC y competencias tecnológicas. En G. Valenti, D. Avaro, y M. Casalet (Eds.), Instituciones, sociedad del conocimiento y mundo del trabajo (pp. 295326). México: FLACSO.

Zuboff, S. (1988). In the Age of the Smart Machine: the Future of Work and Power. New York: Butterworth-Heinemann. 